\title{
Association of systolic blood pressure with atrial fibrillation among treated hypertensive patients
}

\author{
Chaolei Chen ${ }^{1}$, Lin Liu ${ }^{1}$, Yuling Yu ${ }^{1}$, Geng Shen ${ }^{1}$, Jiayi Huang ${ }^{1}$, Yuqing Huang ${ }^{1}$, Kenneth Lo ${ }^{1,2}$, \\ Songtao Tang ${ }^{3 \#}$, Yingqing Feng ${ }^{1 \#}$
}

${ }^{1}$ Department of Cardiology, Hypertension Research Laboratory, Guangdong Provincial Key Laboratory of Coronary Heart Disease Prevention, Guangdong Cardiovascular Institute, Guangdong Provincial People’s Hospital, Guangdong Academy of Medical Sciences, Guangzhou, China; ${ }^{2}$ Centre for Global Cardiometabolic Health, Department of Epidemiology, Brown University, Providence, USA; ${ }^{3}$ Department of Cardiology, Community Health Center of Liaobu County, Dongguan, China

Contributions: (I) Conception and design: ST Tang, YQ Feng, CL Chen; (II) Administrative support: ST Tang, YQ Feng; (III) Provision of study materials or patients: ST Tang, YQ Feng; (IV) Collection and assembly of data: CL Chen, JY Huang, L Liu, YL Yu, G Shen, K Lo, YQ Huang; (V) Data analysis and interpretation: CL Chen, JY Huang, L Liu, YL Yu, G Shen, K Lo, YQ Huang; (VI) Manuscript writing: All authors; (VII) Final approval of manuscript: All authors.

"These authors contributed equally to this work.

Correspondence to: Songtao Tang. Department of Cardiology, Community Health Center of Liaobu County, Dongguan, China. Email: 3038384760@qq.com; Yingqing Feng. Department of Cardiology, Hypertension Research Laboratory, Guangdong Provincial Key Laboratory of Coronary Heart Disease Prevention, Guangdong Cardiovascular Institute, Guangdong Provincial People's Hospital, Guangdong Academy of Medical Sciences, No. 106, Zhongshan Second Road, Yuexiu District, Guangzhou 510080, China. Email: 651792209@qq.com.

Background: Although many studies have suggested the association between elevated blood pressure and atrial fibrillation (AF), how the relationship between systolic blood pressure (SBP) and AF differ by antihypertensive treatment has been unclear. Therefore, this study aimed to explore the relationship between $\mathrm{SBP}$ and $\mathrm{AF}$ in hypertensive patients with or without antihypertensive treatment.

Methods: This was a cross-sectional study that enrolled 7,808 hypertensive patients aged $\geq 18$ years old in 2013 in Guangdong, China. AF was screened and diagnosed by rest 12-lead electrocardiogram (ECG) or by self-reported. Patients were categorized into 5 groups according to a $10 \mathrm{mmHg}$ increment in SBP. We then performed logistic regression and restricted cubic spline regression to evaluate the relationship between SBP and AF.

Results: Out of 7,808 participants (women 52.9\%, mean age 62.3 years), 78 cases of AF were identified. Both univariate and multivariate logistic regression illustrated that SBP associated with a lower chance of AF in all participants when SBP was treated as a continuous variable $(\mathrm{P}<0.05)$ or as a categorical variable $(\mathrm{P}$ for trend $<0.001)$. Similar trend was found in patients with antihypertensive therapy ( $\mathrm{P}$ for trend $<0.001$ ) but not for those without antihypertensive medications.

Conclusions: Our findings suggested that higher SBP is associated with lower likelihood of AF among all hypertensive patients and participants with antihypertensive treatment.

Keywords: Systolic blood pressure; atrial fibrillation (AF); community; hypertension

Submitted Dec 22, 2019. Accepted for publication May 15, 2020.

doi: 10.21037/apm-19-649

View this article at: http://dx.doi.org/10.21037/apm-19-649 


\section{Introduction}

Atrial fibrillation (AF) is a new cardiovascular disease epidemic of the 21 st century (1). According to the 2010 Global Burden of Disease Study, the number of AF patients worldwide was 33.5 million (2). By 2050, there will be 72 million patients with AF just in Asia and 2.9 million AF-associated strokes (3). Previous studies have shown that $\mathrm{AF}$ is independently associated with a 1.5 - and 2-fold increased risk of all-cause mortality in men and women respectively (4-6).

Modifiable risk factors of AF included hypertension, diabetes, myocardial infarction, obesity $(1,7)$. Among all these causes, hypertension is the most important, estimated to account for between $14 \%$ and $22 \%$ of the populationattributable risk which increases to $25 \%$ if borderline hypertension is also included $(8,9)$. Identification, prevention and proper management of blood pressure (BP) is needed for preventing $\mathrm{AF}$.

Meanwhile, both hypertension and AF are long-lasting chronic conditions, requiring repeated measurements for the diagnosis. However, there is limited and conflicting data on the association between $\mathrm{AF}$ and the degree of systolic blood pressure (SBP) control in hypertensive patients, especially in patients receiving antihypertensive treatment. Studies were even fewer in Chinese regions $(10,11)$. In the present study, we investigated the relationship between $\mathrm{SBP}$ and $\mathrm{AF}$ in a group of hypertensive patients in Chinese population with or without antihypertensive medications. We present the following article in accordance with the STROBE reporting checklist (available at http://dx.doi. org/10.21037/apm-19-649).

\section{Methods}

\section{Study population and design}

This population cohort was originated from 8,169 hypertensive patients aged $\geq 18$, who attended annual physical assessment in the community health care center in Liaobu community, Guangdong, China. All participants were enrolled in 2013, diagnosed with essential hypertension and have provided complete sets of data. The participants who did not have data on blood pressure $(n=38)$, blood lipid $(n=245)$, serum creatinine $(n=255)$, demographics $(\mathrm{n}=45)$, and electrocardiogram (ECG) $(\mathrm{n}=71)$ were excluded. Finally, 7808 subjects were included in the analysis (Figure 1). The study complied with the principles outlined in the Declaration of Helsinki (as revised in 2013) and was approved by the institutional medical ethical committee. Written informed consent was obtained from all patients in the study.

\section{Measurement of covariates}

A structured questionnaire was administered by welltrained staff to acquire information on sociodemographic characteristics (including age, sex, smoking, and drinking), medication history [the use of Beta-blockers, calcium channel blockers (CCB), angiotensin-converting enzyme inhibitors (ACEI)/angiotensin-receptor blockers (ARBs), and statins], medical history [ever occurrence of coronary heart disease (CAD), diabetes mellitus (DM), and stroke]. Anthropometric data and biomarkers including body mass index (BMI), SBP, diastolic blood pressure (DBP), total cholesterol (TC), triglyceride (TG), low-density lipoprotein cholesterol (LDL-C), high-density lipoprotein cholesterol (HDL-C), fasting blood glucose (FBG) were measured by physical assessment and laboratory analyses. Blood samples were collected after a fast for at least 8 hours. Estimated glomerular filtration rate (eGFR) was estimated using the simplified equation on the modification of diet in renal disease: $186 \times(\mathrm{Scr})-1.154 \times($ age $)-0.203 \times(0.724$ if female) where Scr was serum creatinine $(\mathrm{mg} / \mathrm{dL})(12)$. Participants were considered to have diagnosed DM if they had previously been diagnosed by a registered medical practitioner, and/or use of hypoglycemic drugs within 2 weeks, and/or with baseline FBG $\geq 7.0 \mathrm{mmol} / \mathrm{L}$. The product of QRS duration was multiplied by the Cornell voltage combination $\left[R_{a V L}+S_{V 3}\right.$, with 6 mm added in women $(13,14)]$ higher than $2,440 \mathrm{~mm} \times$ msec or Sokolow-Lyon voltage $\left(\mathrm{S}_{\mathrm{V} 1}+\mathrm{RV}_{5 / 6}\right)$ higher than $38 \mathrm{~mm}$ (15) on a screening ECG were used to identify left ventricular hypertrophy (LVH).

\section{Blood pressure (BP) measurement}

BP measurement was obtained by trained examiners in the office using the Omron HBP-1100u professional portable BP monitor (Japan) on the right arm. BP was measured twice after a standard protocol and the average of two readings was calculated. Hypertension was defined as SBP $\geq 140 \mathrm{mmHg}$, and/or DBP $\geq 90 \mathrm{mmHg}$, and/or use of antihypertensive medicine within 2 weeks, according to 2010 Chinese guidelines for the management of hypertension (16). 


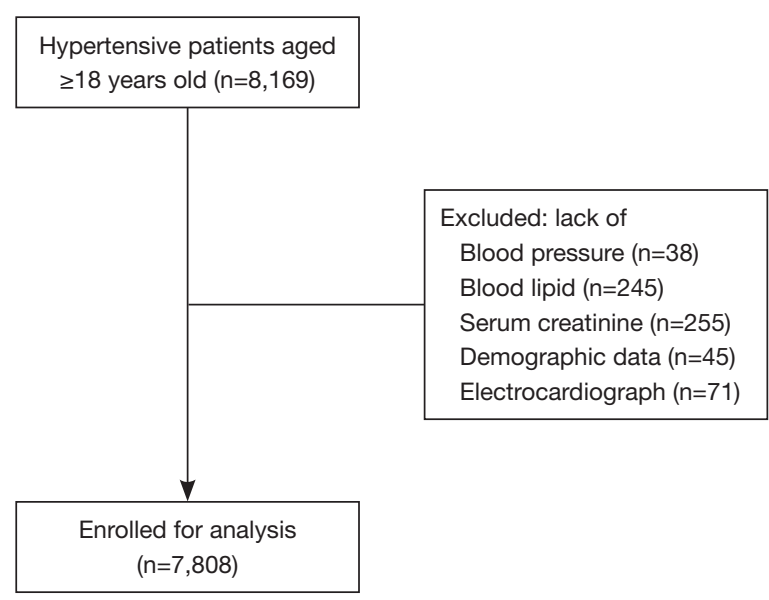

Figure 1 Flow chart of the study participants.

\section{ECG recording and definition of $A F$}

ECG was obtained from standard resting 12-lead recordings at a paper speed of $25 \mathrm{~mm} / \mathrm{s}$ and calibration of $1 \mathrm{mV}$ per $10 \mathrm{~mm}$ and was collected from all subjects at baseline. Participants were considered $\mathrm{AF}$ if $\mathrm{AF}$ rhythm was captured in ECG. AF rhythm was defined as (I) irregular R-R intervals, (II) absence of distinct repeating $\mathrm{P}$ waves, (III) irregular atrial activity show on ECG, according to the 2014 ACC/AHA/HRS Guideline for the Management of Patients With Atrial Fibrillation (17). Those who were not found to have AF on ECG, but had previous medical records from qualified hospital(s) or any prior ECG record(s) of $\mathrm{AF}$ episode(s) were also defined as having $\mathrm{AF}$ (known $\mathrm{AF}$ in sinus rhythm). All the ECG results were identified and diagnosed by qualified practitioners.

\section{Statistical analysis}

Data were presented as mean \pm standard deviation for continuous variables and as proportions for categorical variables. SBP levels were stratified taking $10 \mathrm{mmHg}$ as the interval (<120, 120-130, 130-140, 140-150, $\geq 150 \mathrm{mmHg})$. The one-way ANOVA, Kruskal-Wallis $\mathrm{H}$ test and chisquare test were used to detect any significant differences between subgroups. The association between SBP and $\mathrm{AF}$ was analyzed using logistic regression analysis, with results reported as odds ratio (OR) and $95 \%$ confidence intervals (CIs). Univariate logistic regression was performed to identify significant predictors of AF. The multivariate models included significant predictors that remained significant in multivariate analyses using stepwise forward regression. Besides, we simultaneously showed the results from unadjusted, minimally adjusted and fully adjusted analyses. Fully adjusted models included age, sex, smoking, drinking, DM, CAD, stroke, $\mathrm{LVH}$, antihypertensive medication, DBP, BMI, FBG, eGFR, TC, TG, LDL-C, and HDL-C. Finally, when SBP was analyzed as a continuous variable, restricted cubic spline regression was also applied to identify any association between SBP and AF. A two-tailed $\mathrm{P}$ value of $<0.05$ was required for statistical significance. Statistical analyses were performed using $\mathrm{R}$ version 3.3.2 (R Foundation for Statistical Computing, Vienna, Austria).

\section{Results}

\section{Demographic characteristics}

After applying exclusion criteria, 7,808 participants with a mean age of 62.3 years old were included, with 3,678 (47.1\%) men and 78 cases of AF being identified. The baseline characteristics of the participants were presented in Table 1 and Table 2. In brief, participants with diagnosed AF appeared to be older, had lower levels of SBP, DBP, TG, TC, and LDL-C, had more use of Beta-blockers, and were more likely to have $\mathrm{CAD}$ compared to those without $\mathrm{AF}$ (all $\mathrm{P}<0.01$ ) (Table 1). There were significant differences in age, BMI, SBP, DBP, eGFR, FBG, TC, TG, LDL-C, HDL-C, $\mathrm{DM}, \mathrm{LVH}$, and the use of antihypertensive drugs such as $\mathrm{CCB}$, ACEI, and ARB among the five groups (all $\mathrm{P}<0.05$ ) (Table 2).

\section{Association of SBP with AF in all participants}

Table 3 summarized the results of the relationship between $\mathrm{SBP}$ and $\mathrm{AF}$ among all patients using logistic regression analysis. When we analyzed SBP as a continuous variable, univariate logistic regression illustrated that $\mathrm{SBP}$ was significantly inversely associated with $\mathrm{AF}(\mathrm{P}<0.01)$. In multivariate analysis, the relationship between $\mathrm{SBP}$ and $\mathrm{AF}$ prevalence was also significant (OR per $10-\mathrm{mmHg}$ increase 0.74, 95\% CI: 0.66-0.90, $\mathrm{P}<0.01$ ) (Table 3 and Figure 2). When SBP was treated as a categorical variable, multivariate logistic regression analysis showed that the ORs for AF, using the first group Q1 (SBP $<120 \mathrm{mmHg}$ ) as reference, were 0.69 (95\% CI: $0.38-1.24, \mathrm{P}=0.2158), 0.36$ (95\% CI: 0.18-0.71, $\mathrm{P}=0.0033$ ), 0.42 (95\% CI: 0.19-0.92, $\mathrm{P}=0.0295$ ), and 0.24 (95\% CI: $0.09-0.64, \mathrm{P}=0.0047)$ from $\mathrm{Q} 2$ to $\mathrm{Q} 5$, respectively ( $\mathrm{P}$ for trend $<0.001$ ) (Table 3 and Figure 3). 
Table 1 Baseline characteristics of study participants with and without atrial fibrillation

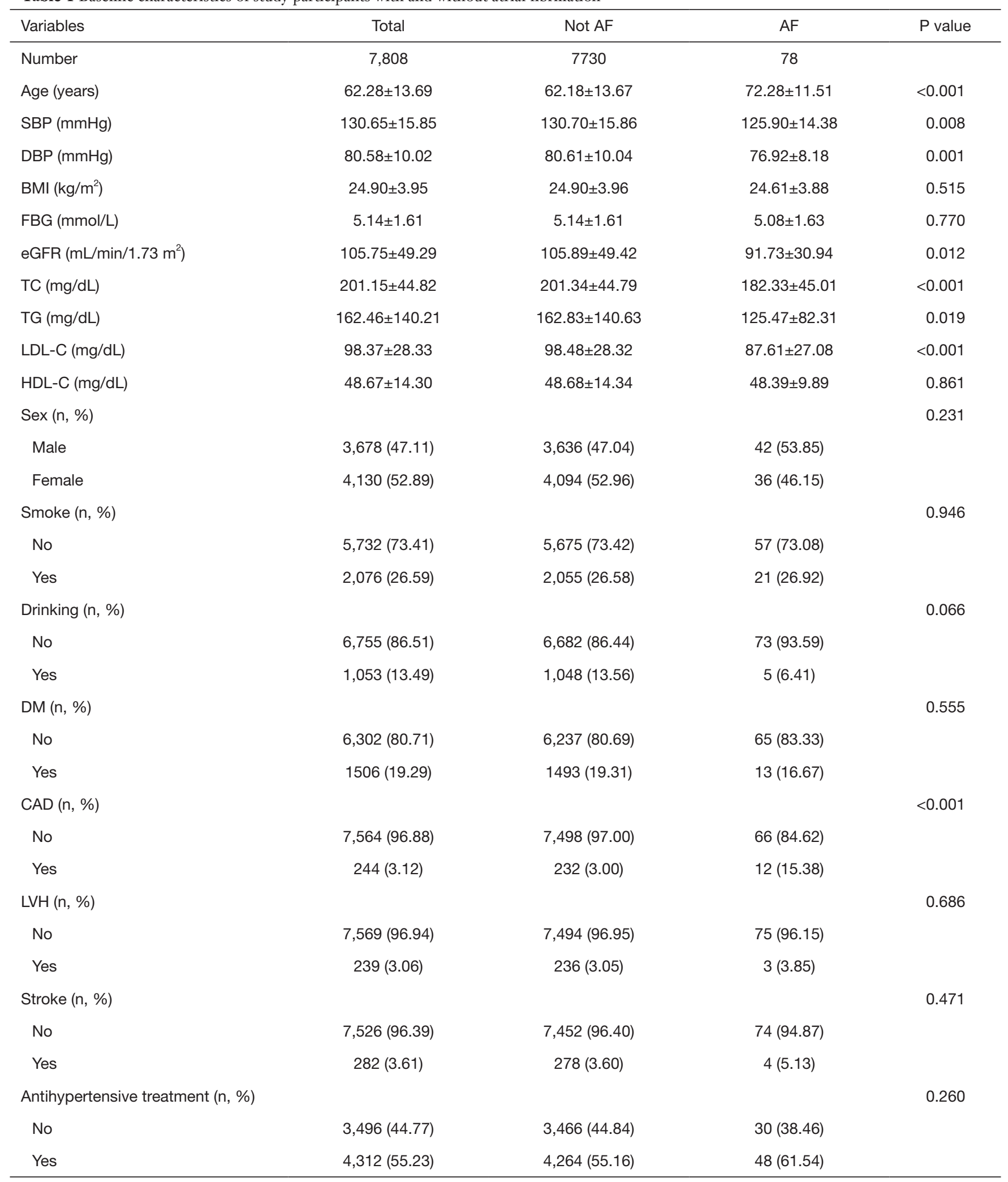

Table 1 (continued) 
Table 1 (continued)

\begin{tabular}{|c|c|c|c|c|}
\hline Variables & Total & Not AF & $\mathrm{AF}$ & $\mathrm{P}$ value \\
\hline No & $7,171(91.84)$ & 7,111 (91.99) & 60 (76.92) & \\
\hline Yes & $637(8.16)$ & $619(8.01)$ & $18(23.08)$ & \\
\hline CCB (n, \%) & & & & 0.923 \\
\hline Yes & 2,463 (31.54) & $2,438(31.54)$ & 25 (32.05) & \\
\hline ACEl/ARB (n, \%) & & & & 0.884 \\
\hline No & 4,441 (56.88) & 4,396 (56.87) & $45(57.69)$ & \\
\hline Yes & 3,367 (43.12) & 3,334 (43.13) & 33 (42.31) & \\
\hline Yes & 1,196 (15.32) & $1,175(15.20)$ & 21 (26.92) & \\
\hline
\end{tabular}

SBP, systolic blood pressure; DBP, diastolic blood pressure; BMI, body mass index; FBG, fasting blood glucose; eGFR, estimated glomerular filtration rate; TC, total cholesterol; TG, triglyceride; LDL-C, low-density lipoprotein cholesterol; HDL-C, high-density lipoprotein cholesterol; DM, diabetes mellitus; CAD, coronary artery disease; LVH, left ventricular hypertrophy; CCB, calcium channel blocker; ACEI, angiotensin enzyme inhibitor; ARB, angiotensin receptor blocker.

Table 2 Baseline characteristics of study participants by systolic blood pressure categories

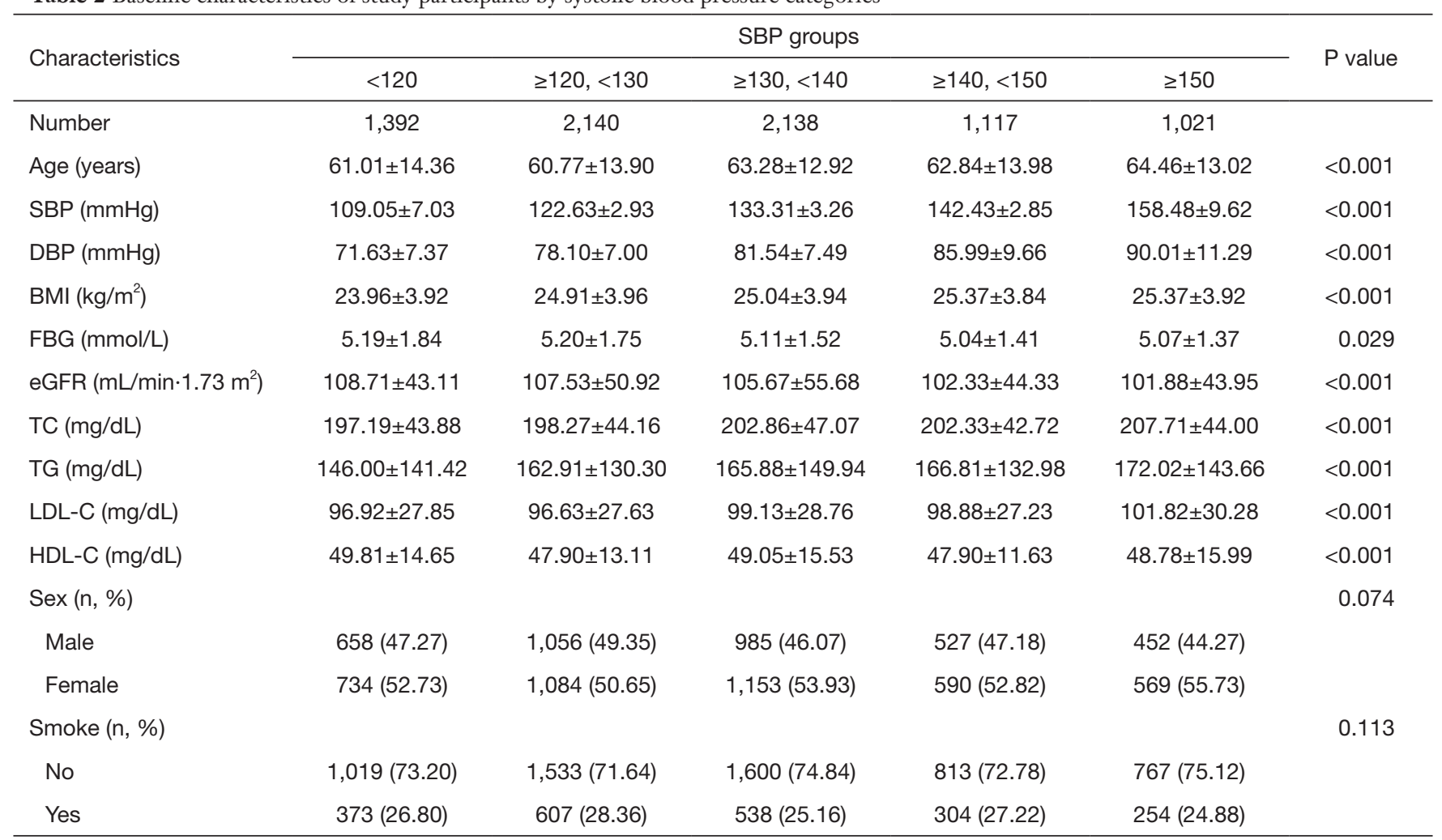

Table 2 (continued) 
Table 2 (continued)

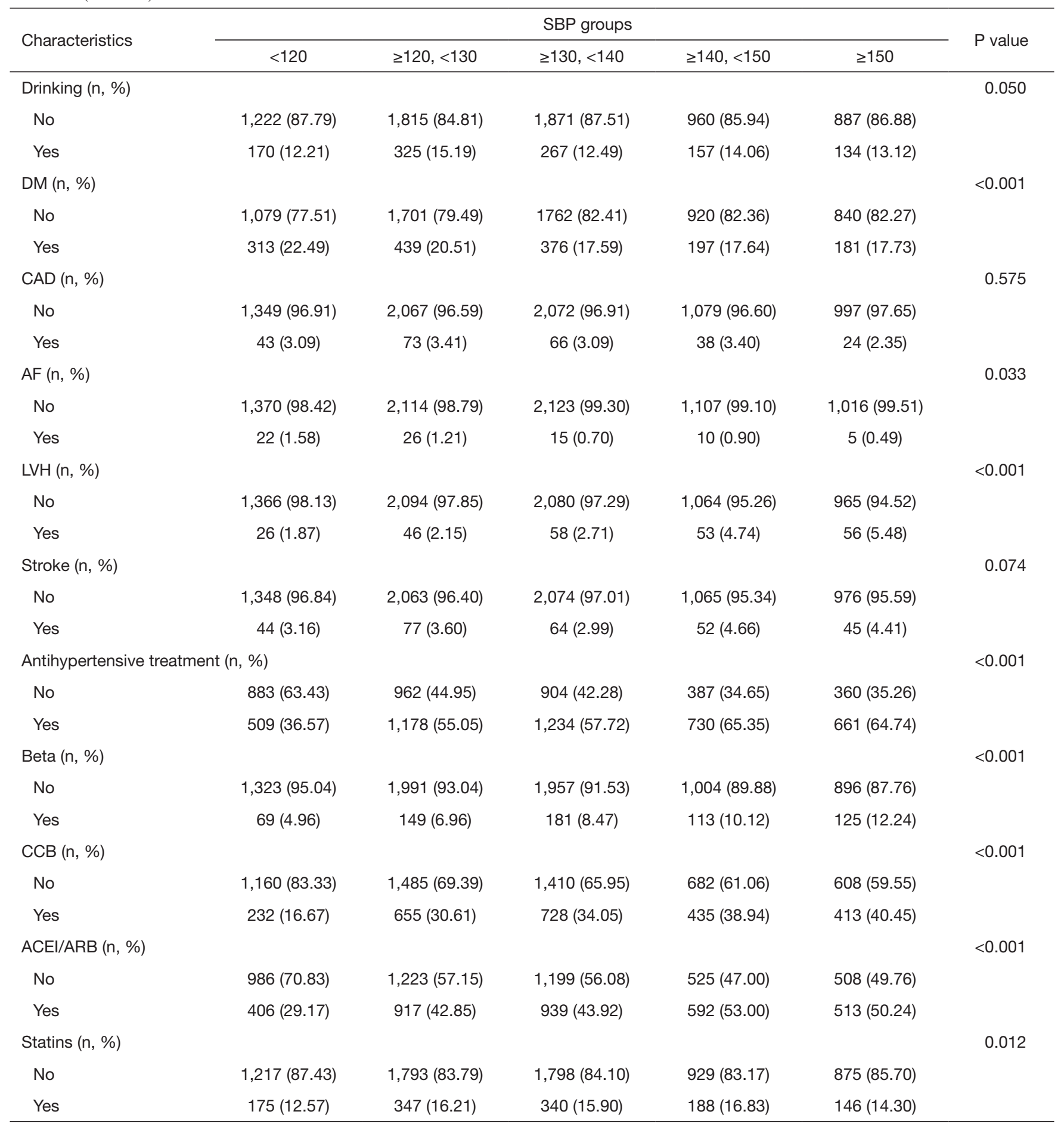

SBP, systolic blood pressure; DBP, diastolic blood pressure; BMI, body mass index; FBG, fasting blood glucose; eGFR, estimated glomerular filtration rate; TC, total cholesterol; TG, triglyceride; LDL-C, low-density lipoprotein cholesterol; HDL-C, high-density lipoprotein cholesterol; DM, diabetes mellitus; CAD, coronary artery disease; LVH, left ventricular hypertrophy; CCB, calcium channel blocker; ACEI, angiotensin enzyme inhibitor; ARB, angiotensin receptor blocker. 
Table 3 Association of systolic blood pressure with atrial fibrillation in the overall population

\begin{tabular}{|c|c|c|c|c|c|c|}
\hline Exposure & \multicolumn{2}{|c|}{ Univariate } & \multicolumn{2}{|c|}{ Adjust I } & \multicolumn{2}{|c|}{ Adjust II } \\
\hline SBP (per 10 mmHg change) & $0.82(0.74,0.90)$ & 0.0075 & $0.74(0.66-0.90)$ & 0.0007 & $0.74(0.66-0.90)$ & 0.0013 \\
\hline \multicolumn{7}{|l|}{ SBP groups } \\
\hline$<120$ & 1.0 & & 1.0 & & 1.0 & \\
\hline$\geq 130,<140$ & $0.44(0.23-0.85)$ & 0.0147 & $0.38(0.19-0.73)$ & 0.0040 & $0.36(0.18-0.71)$ & 0.0033 \\
\hline$\geq 140,<150$ & $0.56(0.27-1.19)$ & 0.1336 & $0.45(0.21-0.96)$ & 0.0402 & $0.42(0.19-0.92)$ & 0.0295 \\
\hline$\geq 150$ & $0.31(0.12-0.81)$ & 0.0174 & $0.24(0.09-0.63)$ & 0.0040 & $0.24(0.09-0.64)$ & 0.0047 \\
\hline$P$ for trend & $<0.001$ & & $<0.001$ & & $<0.001$ & \\
\hline
\end{tabular}

Adjust I model: Adjusted for age, sex, and BMI. Adjust II model: Adjusted for age, sex, smoke, drinking, DM, CAD, LVH, stroke, statins, BMI, FBG, eGFR, TC, TG, LDL-C, HDL-C, and antihypertensive treatment, including beta-blockers, CCB, and ACEI/ARBs. OR, odds ratio; $\mathrm{Cl}$, confidence interval; SBP, systolic blood pressure; $\mathrm{BMI}$, body mass index; FBG, fasting blood glucose; eGFR, estimated glomerular filtration rate; TC, total cholesterol; TG, triglyceride; LDL-C, low-density lipoprotein cholesterol; HDL-C, high-density lipoprotein cholesterol; DM, diabetes mellitus; CAD, coronary artery disease; LVH, left ventricular hypertrophy; CCB, calcium channel blocker; ACEI, angiotensin enzyme inhibitor; ARB, angiotensin receptor blocker.

A

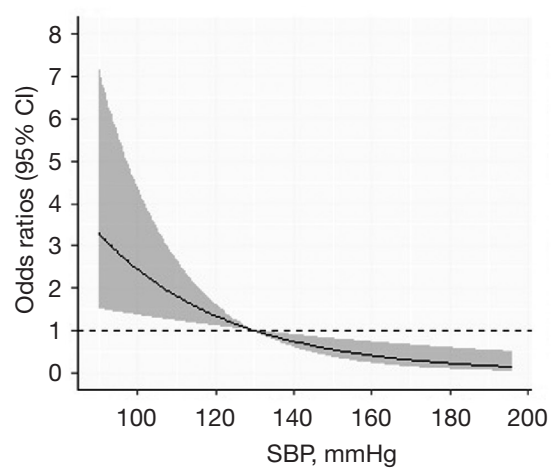

B

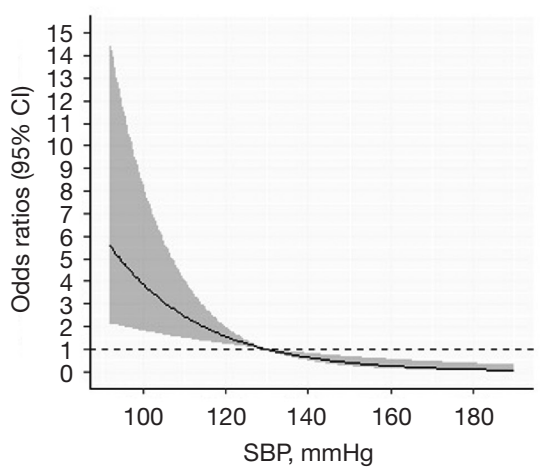

C

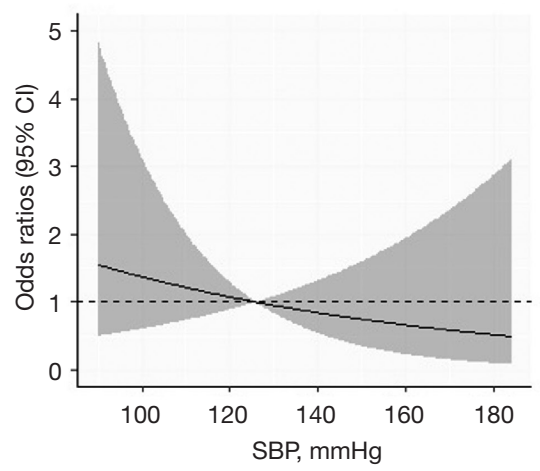

Figure 2 Nature of the relationship between systolic blood pressure and atrial fibrillation. (A) All patients, adjust for age, sex, smoke, drinking, DM, CAD, LVH, stroke, antihypertensive treatment, statins, BMI, FBG, eGFR, TC, TG, LDL-C, HDL-C; (B) treated patients, adjust for age, sex, smoke, drinking, DM, CAD, LVH, stroke, Statin, BMI, FBG, eGFR, TC, TG, LDL-C, HDL-C; (C) untreated patients, adjust for age, sex, smoke, drinking, DM, CAD, LVH, stroke, Statin, BMI, FBG, eGFR, TC, TG, LDL-C, HDL-C. SBP, systolic blood pressure; BMI, body mass index; FBG, fasting blood glucose; eGFR, estimated glomerular filtration rate; TC, total cholesterol; TG, triglyceride; LDL-C, low-density lipoprotein cholesterol; HDL-C, high-density lipoprotein cholesterol; DM, diabetes mellitus; CAD, coronary artery disease; LVH, left ventricular hypertrophy.

\section{Subgroup analysis}

To further explore the relationship between SBP and AF among treated and untreated patients, we divided participants into two groups by the use of anti-hypertensive treatments. The results were revealed in Table 4 and Figure 3, and the linear relationship between $\mathrm{SBP}$ and $\mathrm{AF}$ was shown in Figure 2. In summary, higher SBP levels were associated with lower AF prevalence among treated patients (P for trend $<0.001$ ), but not for patients not receiving antihypertensive treatment. 


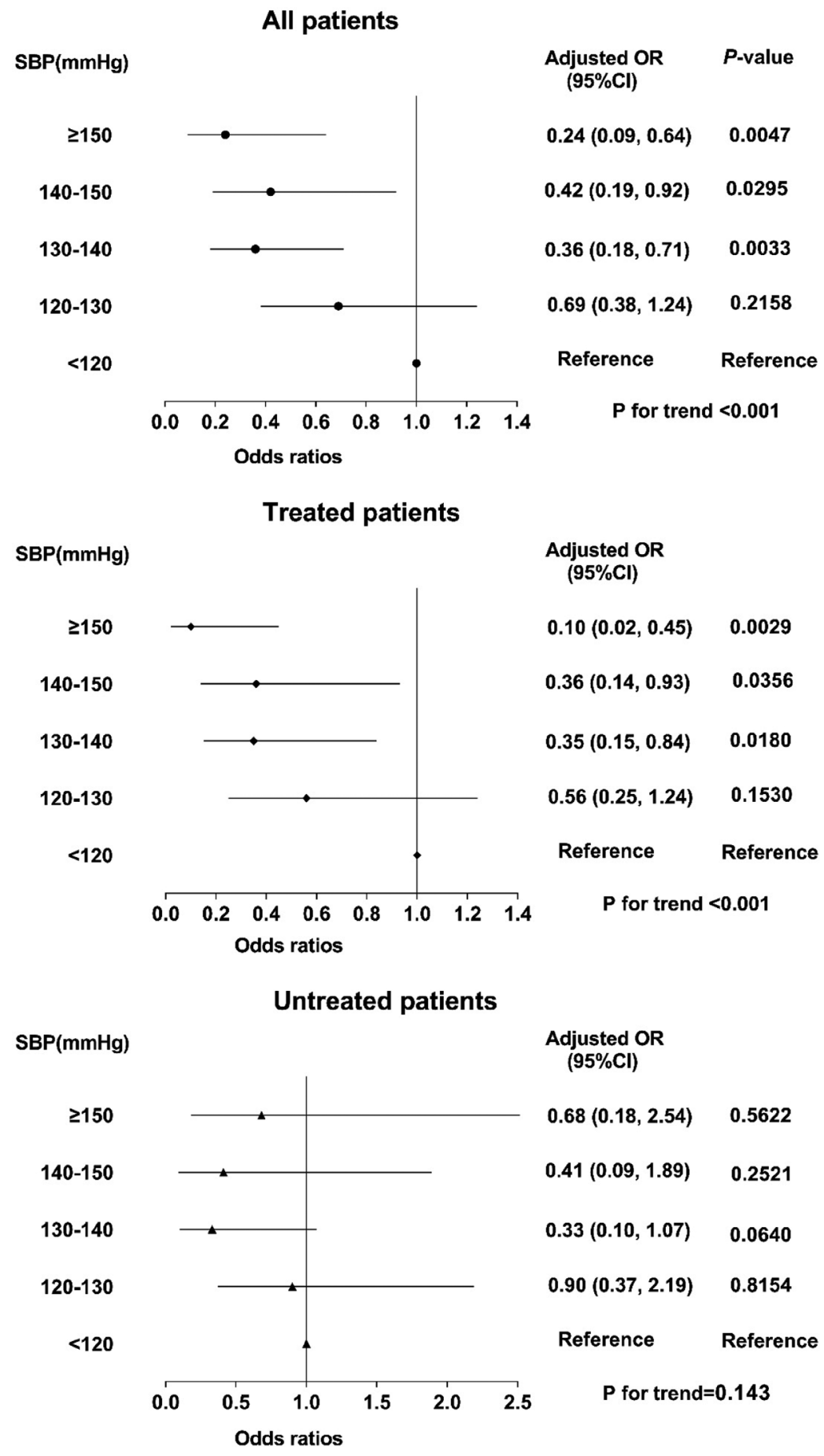

Figure 3 Association of systolic blood pressure with atrial fibrillation in the overall population and subgroups. All patients: adjust for age, sex, smoke, drinking, DM, CAD, LVH, stroke, antihypertensive treatment, statins, BMI, FBG, eGFR, TC, TG, LDL-C, HDL-C; treated and untreated patients: adjust for: age, sex, smoke, drinking, DM, CAD, LVH, stroke, Statin, BMI, FBG, eGFR, TC, TG, LDL-C, HDL-C. OR, odds ratio; CI, confidence interval; SBP, systolic blood pressure; BMI, body mass index; FBG, fasting blood glucose; eGFR, estimated glomerular filtration rate; TC, total cholesterol; TG, triglyceride; LDL-C, low-density lipoprotein cholesterol; HDL-C, highdensity lipoprotein cholesterol; DM, diabetes mellitus; CAD, coronary artery disease; LVH, left ventricular hypertrophy. 
Table 4 Subgroup analyses according to antihypertensive treatment

\begin{tabular}{|c|c|c|c|c|}
\hline Exposure & \multicolumn{2}{|c|}{ Untreated } & \multicolumn{2}{|c|}{ Treated } \\
\hline \multicolumn{5}{|l|}{ Non-adjusted } \\
\hline SBP (per 10 mmHg change) & $0.90(0.74-1.10)$ & 0.3228 & $0.74(0.60-0.90)$ & 0.0025 \\
\hline \multicolumn{5}{|l|}{ SBP groups } \\
\hline$\geq 120,<130$ & $0.83(0.35-1.97)$ & 0.6769 & $0.62(0.29-1.35)$ & 0.2319 \\
\hline$\geq 130,<140$ & $0.35(0.11-1.11)$ & 0.0749 & $0.41(0.18-0.95)$ & 0.0365 \\
\hline$\geq 140,<150$ & $0.41(0.09-1.87)$ & 0.2499 & $0.50(0.20-1.26)$ & 0.1407 \\
\hline$\geq 150$ & $0.67(0.18-2.40)$ & 0.5347 & $0.14(0.03-0.62)$ & 0.0100 \\
\hline \multicolumn{5}{|l|}{ Adjust I } \\
\hline SBP (per 10 mmHg change) & $0.82(0.66-1.10)$ & 0.1663 & $0.66(0.54-0.82)$ & 0.0002 \\
\hline \multicolumn{5}{|l|}{ SBP groups } \\
\hline$<120$ & 1.0 & & 1.0 & \\
\hline$\geq 120,<130$ & $0.78(0.33-1.85)$ & 0.5693 & $0.56(0.25-1.23)$ & 0.1467 \\
\hline$\geq 130,<140$ & $0.29(0.09-0.93)$ & 0.0369 & $0.34(0.15-0.81)$ & 0.0147 \\
\hline$\geq 140,<150$ & $0.35(0.08-1.59)$ & 0.1725 & $0.35(0.14-0.89)$ & 0.0268 \\
\hline$\geq 150$ & $0.55(0.15-2.00)$ & 0.3633 & $0.09(0.02-0.42)$ & 0.0021 \\
\hline$P$ for trend & 0.072 & & & $<0.001$ \\
\hline$\geq 130,<140$ & $0.33(0.10-1.07)$ & 0.0640 & $0.35(0.15-0.84)$ & 0.0180 \\
\hline$\geq 140,<150$ & $0.41(0.09-1.89)$ & 0.2521 & $0.36(0.14-0.93)$ & 0.0356 \\
\hline$\geq 150$ & $0.68(0.18-2.54)$ & 0.5622 & $0.10(0.02-0.45)$ & 0.0029 \\
\hline$P$ for trend & 0.143 & & & $<0.001$ \\
\hline
\end{tabular}

The association between SBP and AF in subgroups was estimated using odds ratio (OR), 95\% confidence interval (Cl) and $\mathrm{P}$ value. Nonadjusted model: adjusted for none. Adjust I model: adjusted for age, sex, and BMI. Adjust II model: adjust for age, sex, smoke, drinking, DM, CAD, LVH, stroke, statins, BMI, FBG, eGFR, TC, TG, LDL-C, and HDL-C. SBP, systolic blood pressure; BMI, body mass index; FBG, fasting blood glucose; eGFR, estimated glomerular filtration rate; TC, total cholesterol; TG, triglyceride; LDL-C, low-density lipoprotein cholesterol; HDL-C, high-density lipoprotein cholesterol; DM, diabetes mellitus; CAD, coronary artery disease; LVH, left ventricular hypertrophy.

\section{Discussion}

In the present study, SBP was significantly associated with AF prevalence in all hypertensive participants and for patients with antihypertensive therapy ( $\mathrm{P}$ for trend $<0.001$ ).
Our findings were inconsistent with some previous studies. When looking into two long-term population studies, SBP associated with a higher risk of incident $\mathrm{AF}$ in both men and women $(18,19)$. Results from 2014 healthy 
middle-aged men enrolled in the Norwegian study (19) and have been followed up for a median period of 35 years showed that men with baseline SBP $\geq 140 \mathrm{mmHg}$ and for those with SBP at $128-138 \mathrm{mmHg}$ had 1.60 -fold (95\% CI: 1.15-2.21) and 1.50-fold (95\% CI: 1.10-2.03) risk of AF, respectively, compared with men with $\mathrm{SBP}<128 \mathrm{mmHg}$. Similar results were observed in the Women's Health Study (18) in 34,221 women with a median follow-up period of 14 years. Compared with optimal SBP, the hazard ratios (HRs) of incident AF for high-normal SBP and stage 1 and stage 2 or 3 systolic hypertension were 1.28 (95\% CI: 1.00-1.63), 1.56 (95\% CI: $1.22-2.01$ ), and 2.74 (95\% CI: 1.77-4.22), respectively.

Moreover, the REGARDS study (Reasons for Geographic and Racial Differences in Stroke) did not observe any significant differences in the prevalence of $\mathrm{AF}$ in relation to SBP levels in a large mixed population of normotensive and hypertensive individuals (20). Besides, in line with our findings, a cross-sectional study (10) enrolled 6,966 elderly residents and suggested a negative relationship between $\mathrm{SBP}$ and prevalent $\mathrm{AF}$ (per $10 \mathrm{mmHg}$ increase, $\mathrm{OR}=0.79$, $95 \%$ CI: $0.71-0.88, \mathrm{P}<0.0001)$. Similar trend was observed in one previous study which enrolled 17003 older patients initiating hemodialysis with 5 years of follow-up (21). Reducing SBP for $10 \mathrm{mmHg}$ was significantly associated with a higher HR for AF (HR=1.12, 95\% CI: 1.10-1.14). These studies suggested that large-scale prospective studies or randomized controlled trials were needed to verify the association between SBP and AF.

Patients with antihypertensive treatment comprised of a pre-specified subgroup of special interest in the present study. Consistent with the overall finding, SBP was also associated with a lower rate of prevalent $\mathrm{AF}$ in patients who were pharmacologically treated for hypertension. The Losartan Intervention For Endpoint reduction in hypertension (LIFE) study found that among patients with hypertension and ECG-LVH, achieving SBP $\leq 130 \mathrm{mmHg}$ was associated with a $40 \%$ lower risk (95\% CI: $18-55 \%$ ) of new AF (22). The preventive effect of antihypertensive therapy was also confirmed in another analysis of the LIFE study in a subgroup of patients with isolated systolic hypertension (23). However, the conclusions could not be generalized to hypertensive patients at lower risk because using ECG-LVH criteria to select patients for LIFE increased the baseline risk of the population. Among all the patients in our study, only $3 \%$ had LVH at baseline. Besides, a J-shaped relationship between SBP and AF was found in a case-control study of patients undergoing treatment for hypertension (24).

The mechanisms behind how SBP may physiologically relate with prevalent AF remains unclear. In general, Generally, hypertension promotes the remodeling of the heart's structure and function after electrophysiological changes in the left atrium, and then lead to the development of AF by increasing the heart's pulsating load and increasing the size of the left atrium (25). Besides, hypertension and $\mathrm{LVH}$ are the incentives for the development of AF, causing excessive sympathetic nerve activity. The result is an overreaction to stress-stimulated adrenaline, which leads to arrhythmias such as AF (26). However, the relationship between hypertension and AF is complicated because they share common risk factors. Apart from traditional risk factors, a recent study showed the combined effect of duration of hypertension and body weight status on the risk of new-onset AF, concluding that the highest risk for $\mathrm{AF}$ existed in patients with obesity and hypertension onset in no less than 5 years (27). Even though the onset duration of hypertension was not available in our study, the main findings remained significant after adjusting for many risk factors for AF, which increased the reliability of our research findings. A potential explanation of the negative correlation of SBP and AF is reverse causality. Low SBP may indeed increase the risk of AF via chronic coronary ischemia, myocardial proliferation and fibrosis induced by inadequate coronary perfusion which leads to the development of AF. On the other hand, low SBP may be the result of AF or AF-related cardiac structural and functional abnormalities $(10,28)$. Another explanation may be that individuals with higher SBP levels are more likely to receive better clinical care and more usage of antihypertensive medications, which plays an important role in the mutually influenced relationship between hypertension and $\mathrm{AF}$.

Worthy to mention, previous studies have proved that inhibition of renin-angiotensin-aldosterone system (RAAS) with ACEI or ARBs compared with $\beta$-blockers and diuretics associated with a reduced risk of $\mathrm{AF}(1,17)$. In our study, the relationship between $\mathrm{SBP}$ and $\mathrm{AF}$ remained significant after adjusting for antihypertensive treatment including ACEI and ARBs. Due to the insufficient data on the use of diuretics, we could not examine the possible relationship between hypovolemia and $\mathrm{AF}$.

Some limitations should be taken into consideration to make cautious interpretation of our study. First, our study did not elucidate causation due to the cross-sectional design. Second, in the present analysis, AF cases were ascertained using ECGs. However, the patients that did not perform 
dynamic ECG detection, the true prevalence may have been underestimated by missing possible cases of paroxysmal $\mathrm{AF}$, like all similar studies on AF. Current ESH/ESC guidelines recommend that ECG monitoring should be performed to all hypertensive patients, so there is a need for more extended and reliable AF screening in the future. Third, the absence of data on the left atrial size in the vast majority of patients might preclude a meaningful evaluation of whether the relationship of $\mathrm{SBP}$ to prevalent $\mathrm{AF}$ could be in part explained by differences in left atrial size. Finally, we recruited patients from a single center in China, therefore the results might not be extrapolated to other populations and ethnic groups.

\section{Conclusions}

Our findings suggested that higher SBP is associated with lower likelihood of AF among all hypertensive patients and participants with antihypertensive treatment. The findings may allow clinicians to provide better-personalized plans for disease management. More large-scaled prospective studies are needed to further verify our findings.

\section{Acknowledgments}

Funding: This work was supported by the Natural Science Foundation of Guangdong Province (No. 2020A1515010738), the Science and Technology Program of Guangzhou (No. 201604020143, No. 201604020018, No. 201604020186 , No. 201510010254 , and No. 201803040012), and the National Key Research and Development Program of China (No. 2017FYC1307603, No. 2016YFC1301305), the Key Area R\&D Program of Guangdong Province (No. 2019B020227005).

\section{Footnote}

Reporting Checklist: The authors have completed the STROBE reporting checklist. Available at http://dx.doi. org/10.21037/apm-19-649

Data Sharing Statement: Available at http://dx.doi. org/10.21037/apm-19-649

Peer Review File: Available at http://dx.doi.org/10.21037/ apm-19-649

Conflicts of Interest: All authors have completed the ICMJE uniform disclosure form (available at http://dx.doi. org/10.21037/apm-19-649). The authors have no conflicts of interest to declare.

Ethical Statement: The authors are accountable for all aspects of the work in ensuring that questions related to the accuracy or integrity of any part of the work are appropriately investigated and resolved. The study was conducted in accordance with the Declaration of Helsinki (as revised in 2013). The study was approved by the institutional ethics committee of the Guangdong Provincial People's Hospital (No. GDREC2012143H). Written informed consent was obtained from all patients in the study. A copy of the written consent is available for review by the Editor-in-Chief of this journal.

Open Access Statement: This is an Open Access article distributed in accordance with the Creative Commons Attribution-NonCommercial-NoDerivs 4.0 International License (CC BY-NC-ND 4.0), which permits the noncommercial replication and distribution of the article with the strict proviso that no changes or edits are made and the original work is properly cited (including links to both the formal publication through the relevant DOI and the license). See: https://creativecommons.org/licenses/by-nc$\mathrm{nd} / 4.0 /$.

\section{References}

1. Kirchhof P, Benussi S, Kotecha D, et al. 2016 ESC Guidelines for the management of atrial fibrillation developed in collaboration with EACTS. Eur Heart J 2016;37:2893-962.

2. Chugh SS, Havmoeller R, Narayanan K, et al. Worldwide epidemiology of atrial fibrillation: a Global Burden of Disease 2010 Study. Circulation 2014;129:837-47.

3. Rahman F, Kwan GF, Benjamin EJ. Global epidemiology of atrial fibrillation. Nat Rev Cardiol 2016;13:501.

4. Ruddox V, Sandven I, Munkhaugen J, et al. Atrial fibrillation and the risk for myocardial infarction, all-cause mortality and heart failure: A systematic review and metaanalysis. Eur J Prev Cardiol 2017;24:1555-66.

5. Magnussen C, Niiranen TJ, Ojeda FM, et al. Sex Differences and Similarities in Atrial Fibrillation Epidemiology, Risk Factors, and Mortality in Community Cohorts: Results From the BiomarCaRE Consortium (Biomarker for Cardiovascular Risk Assessment in Europe). Circulation 2017;136:1588-97.

6. Odutayo A, Wong CX, Hsiao AJ, et al. Atrial fibrillation 
and risks of cardiovascular disease, renal disease, and death: systematic review and meta-analysis. BMJ 2016;354:i4482.

7. Calkins H, Hindricks G, Cappato R, et al. 2017 HRS/ EHRA/ECAS/APHRS/SOLAECE expert consensus statement on catheter and surgical ablation of atrial fibrillation: Executive summary. Europace 2018;20:157-208.

8. Lee SS, Ae Kong K, Kim D, et al. Clinical implication of an impaired fasting glucose and prehypertension related to new onset atrial fibrillation in a healthy Asian population without underlying disease: a nationwide cohort study in Korea. Eur Heart J 2017;38:2599-607.

9. Schnabel RB, Yin X, Gona P, et al. 50 year trends in atrial fibrillation prevalence, incidence, risk factors, and mortality in the Framingham Heart Study: a cohort study. Lancet 2015;386:154-62.

10. Chen Y, Huang QF, Sheng CS, et al. Cross-sectional Association Between Blood Pressure Status and Atrial Fibrillation in an Elderly Chinese Population. Am J Hypertens 2019;32:777-85.

11. Yang Y, Han X, Chen Y, et al. Association between modifiable lifestyle and the prevalence of atrial fibrillation in a Chinese population: Based on the cardiovascular health score. Clin Cardiol 2017;40:1061-7.

12. Guo M, Niu JY, Ye XW, et al. Evaluation of various equations for estimating renal function in elderly Chinese patients with type 2 diabetes mellitus. Clin Interv Aging 2017;12:1661-72.

13. Zhang W, Zhou Y, Bai B, et al. Consistency of left ventricular hypertrophy diagnosed by electrocardiography and echocardiography: the Northern Shanghai Study. Clin Interv Aging 2019;14:549-56.

14. Okin PM, Hille DA, Kjeldsen SE, et al. Combining ECG Criteria for Left Ventricular Hypertrophy Improves Risk Prediction in Patients With Hypertension. J Am Heart Assoc 2017;6:e007564.

15. Kim D, Lee GY, Choi JO, et al. Associations of Electrocardiographic Parameters with Left Ventricular Longitudinal Strain and Prognosis in Cardiac Light Chain Amyloidosis. Sci Rep 2019;9:7746.

16. Liu LS. 2010 Chinese guidelines for the management of hypertension. Zhonghua Xin Xue Guan Bing Za Zhi 2011;39:579-615.

17. January CT, Wann LS, Alpert JS, et al. 2014 AHA/ACC/ HRS guideline for the management of patients with atrial fibrillation: executive summary: a report of the American College of Cardiology/American Heart Association Task Force on practice guidelines and the Heart Rhythm Society. Circulation 2014;130:2071-104.
18. Conen D, Tedrow UB, Koplan BA, et al. Influence of systolic and diastolic blood pressure on the risk of incident atrial fibrillation in women. Circulation 2009;119:2146-52.

19. Grundvold I, Skretteberg PT, Liestol K, et al. Upper normal blood pressures predict incident atrial fibrillation in healthy middle-aged men: a 35-year follow-up study. Hypertension 2012;59:198-204.

20. Bhatt H, Gamboa CM, Safford MM, et al. Is there an association between the prevalence of atrial fibrillation and severity and control of hypertension? The REasons for Geographic And Racial Differences in Stroke study. J Am Soc Hypertens 2016;10:578-86.e5.

21. Chang TI, Liu S, Airy M, et al. Blood Pressure and Incident Atrial Fibrillation in Older Patients Initiating Hemodialysis. Clin J Am Soc Nephrol 2019;14:1029-38.

22. Okin PM, Hille DA, Larstorp AC, et al. Effect of lower on-treatment systolic blood pressure on the risk of atrial fibrillation in hypertensive patients. Hypertension 2015;66:368-73.

23. Larstorp ACK, Stokke IM, Kjeldsen SE, et al. Antihypertensive therapy prevents new-onset atrial fibrillation in patients with isolated systolic hypertension: the LIFE study. Blood Press 2019;28:317-26.

24. Thomas MC, Dublin S, Kaplan RC, et al. Blood pressure control and risk of incident atrial fibrillation. Am J Hypertens 2008;21:1111-6.

25. Colussi G, Catena C, Fagotto V, et al. Atrial fibrillation and its complications in arterial hypertension: The potential preventive role of $\omega-3$ polyunsaturated fatty acids. Crit Rev Food Sci Nutr 2019;59:1937-48.

26. Kallistratos MS, Poulimenos LE, Manolis AJ. Atrial fibrillation and arterial hypertension. Pharmacol Res 2018;128:322-6.

27. Kim YG, Han KD, Choi JI, et al. Impact of the Duration and Degree of Hypertension and Body Weight on NewOnset Atrial Fibrillation: A Nationwide Population-Based Study. Hypertension 2019;74:e45-e51.

28. Dzeshka MS, Shantsila A, Shantsila E, et al. Atrial Fibrillation and Hypertension. Hypertension 2017;70:854-61.

Cite this article as: Chen C, Liu L, Yu Y, Shen G, Huang J, Huang Y, Lo K, Tang S, Feng Y. Association of systolic blood pressure with atrial fibrillation among treated hypertensive patients. Ann Palliat Med 2020;9(4):1752-1763. doi: 10.21037/ apm-19-649 